\title{
Physics Results of the LHCf Experiment
}

\author{
Alessia Tricomi on behalf of the LHCf Collaboration* \\ University of Catania and INFN Catania, Italy \\ E-mail: alessia.tricomiect.infn.it
}

The LHCf experiment has been designed to precisely measure very forward neutral particle spectra produced in the high energy hadron-hadron collisions at LHC up to an energy of $14 \mathrm{TeV}$ in the center of mass system. These measurements are of fundamental importance to calibrate the Monte Carlo models widely used in the high energy cosmic ray (HECR) field, up to an equivalent laboratory energy of the order of $10^{17} \mathrm{eV}$.

The experiment has taken data in p-p collisions at $\sqrt{s}=0.9 \mathrm{TeV}, \sqrt{s}=2.76 \mathrm{TeV}$ and $\sqrt{s}=7$ $\mathrm{TeV}$ as well as in $\mathrm{p}-\mathrm{Pb}$ collisions at $\sqrt{s}=5 \mathrm{TeV}$. In this paper the most up-to-date results on the inclusive photon spectra, $\pi^{0}$ and neutron spectra measured by LHCf are reported. Comparison of these spectra with the model expectations and the impact on high energy cosmic ray (HECR) Physics are discussed. In addition, perspectives for future analyses as well as the program for the next data taking period will be discussed.

The European Physical Society Conference on High Energy Physics -EPS-HEP2013

18-24 July 2013

Stockholm, Sweden

${ }^{*}$ Speaker. 


\section{Introduction}

Dedicated extensive air shower experiments are taking data since many years and have strongly contributed to our understanding of High and Ultra High Energy Cosmic (UHECR) Ray Physics. However, the results of these experiments are in some cases not fully in agreement and, in addition, the interpretation of their data in terms of primary cosmic ray properties is strongly affected by the knowledge of the nuclear interactions in the earth's atmosphere. This is true, for instance, for the interpretation of the behaviour of the energy spectrum in the UHE region, in particular the existence of events above the so called GZK cut-off, and the chemical composition of cosmic rays. Indeed, evidence of UHECR, above the GZK cut-off, has been reported for the first time by the AGASA experiment [1]. On the contrary, the results of the HiRes [2] experiment and, more recently, the ones of the Pierre Auger Collaboration [3] and the Telescope Array Collaboration [4] are consistent with the existence of the cut-off. The disagreement among data would be reduced by adjusting the energy scales of the different experiments to account for systematic effects in the determination of the particle energy, that might be due to different detecting techniques. Similar considerations hold for the interpretation of cosmic ray composition since it is directly related to their primary sources. Accelerator experiments validating the interaction model chosen are hence essential. As a matter of fact air shower development is dominated by the forward products of the interaction between the primary particle and the atmosphere. The LHCf experiment at LHC has been specifically designed to study neutral particles produced in high energy hadron-hadron collisions at LHC in the very forward region, covering the $|\eta|>8.3$ pseudo-rapidity region. LHCf has the unique opportunity to take data at energies ranging from $\sqrt{s}=0.9 \mathrm{TeV}$ up to $14 \mathrm{TeV}$, thus extending significantly the energy range up to a region of great interest for high energy cosmic rays, the region between the "knee" and the GZK cut-off. In addition, the possibility to take data in p-Ion run offers a unique way to study also in-medium effect those significantly improvement the available knowledge of the hadronic interaction mechanisms.

\section{The LHCf detector}

The LHCf experiment is composed by two independent position sensitive electromagnetic calorimeters. Each calorimeter (ARM1 and ARM2) has a double tower structure, with the smaller tower located at zero degree collision angle, approximately covering the region with pseudo-rapidity $\eta>10$ and the larger one, approximately covering the region with $8.4<\eta<10$. Four X-Y layers of position sensitive detectors (scintillating fibers in ARM1, silicon micro-strip detectors in ARM2) provide measurements of the transverse profile of the showers. The two tower structure allows to reconstruct the $\pi^{0}$ decaying in two $\gamma_{\mathrm{s}}$, hitting separately the two towers, hence providing a very precise absolute energy calibration of the detectors. In the range $\mathrm{E}>100 \mathrm{GeV}$, the LHCf detectors have energy and position resolutions for electromagnetic showers better than $5 \%$ and $200 \mu \mathrm{m}$, respectively.

The detectors are installed on both side of the ATLAS experiment, $140 \mathrm{~m}$ away from the LHC-IP1 interaction point, inside the zero-degree neutral absorber (Target Neutral Absorber, TAN). Charged particles from the IP are swept away by the inner beam separation dipole before reaching the TAN, 
so that only photons mainly from $\pi^{0}$ decays and neutral hadrons reach the LHCf calorimeters. A detailed description of the LHCf experiment can be found in Ref. [6].

\section{Results of the p-p data taking}

This section summarize some of the results that have been recently published by the LHCf collaboration, concerning the single photon spectra and the $\pi^{0}$ spectra in p-p collisions [7, 8, 9], and shows some preliminary results obtained for the neutron spectra.

\subsection{Measurement of the single photon energy spectra}

The LHCf Collaboration has measured the single photon energy spectrum in proton-proton collisions at $7 \mathrm{TeV}$ [7] and, more recently, at $900 \mathrm{GeV}$ p-p collisions [8]. Here we briefly summarize the main steps of the analysis with special emphasis to possible implication for the calibration of Monte Carlo models used in HECR Physics. For both analyses only a subset of the collected data have been analysed corresponding to an integrated luminosity of $0.68 \mathrm{nb}^{-1}$ and $0.52 \mathrm{nb}^{-1}$ for the ARM1 and ARM2 detectors, respectively, for the $7 \mathrm{TeV}$ data and to an integrated luminosity of 0.30 $\mathrm{nb}^{-1}$ for the $900 \mathrm{GeV}$ analysis. The analysed data have been chosen in a particularly clean and low luminosity fill, to minimize backgrounds, hence reducing the systematics of the measurement.

The main steps of the analysis work-flow are almost identical for the two analysis.

The energy of photons is reconstructed from the signal released by the shower particles in the scintillators, after applying corrections for the non-uniformity of light collection and for particles leaking in and out of the edges of the calorimeter towers. In order to correct for these last two effects, which are rather important due to the limited transverse size of both the calorimetric towers, the transverse impact position of showers provided by the position sensitive detectors is used.

Event produced by neutral hadrons are rejected applying a simple particle identification algorithm based on the longitudinal development of the showers, which is different for electromagnetic and hadronic particles. In addition, for the $7 \mathrm{TeV}$ analysis, thanks to the information provided by the position sensitive detectors, events with more than one shower inside the same tower (multi-hit) are rejected, while for the $900 \mathrm{GeV}$ analysis the number of multi-particle events is negligible hence the multi-hit rejection is not applied. In order to combine the spectra measured by ARM1 and ARM2, which have different geometrical configurations, in these analyses only events detected in a common pseudo-rapidity and azimuthal range are selected: $\eta>10.94$ and $8.81<\eta<8.99$, for the small and large towers, respectively, for $7 \mathrm{TeV}$ analysis and $\eta>10.15$ and $8.77<\eta<9.46$, for the small and large towers, respectively, for $900 \mathrm{GeV}$ analysis.

The single $\gamma$ spectra measured by LHCf in the two pseudo-rapidity regions for $7 \mathrm{TeV}$ and 900 $\mathrm{GeV}$ p-p collisions, respectively, have been compared with results predicted by $\mathrm{MC}$ simulations using different models. Figure 1 shows the ratio between data and MC predictions using different models: DPMJET III-3.04 [10], QGSJET II-03 [11], SIBYLL 2.1 [12], EPOS 1.9 [13] and PYTHIA 8.145 [14]. Statical errors and systematic uncertainties are also plotted. A careful study of systematic uncertainties has been done and conservative estimates have been taken into account. Further details can be found in Ref. [7, 8]. As can be seen from Fig. 1, a clear discrepancy between the experimental results and the predictions of the models in particular in the high energy region is 
present. The impact of such results in the tuning of MC code is now under investigation with the help of code developers.
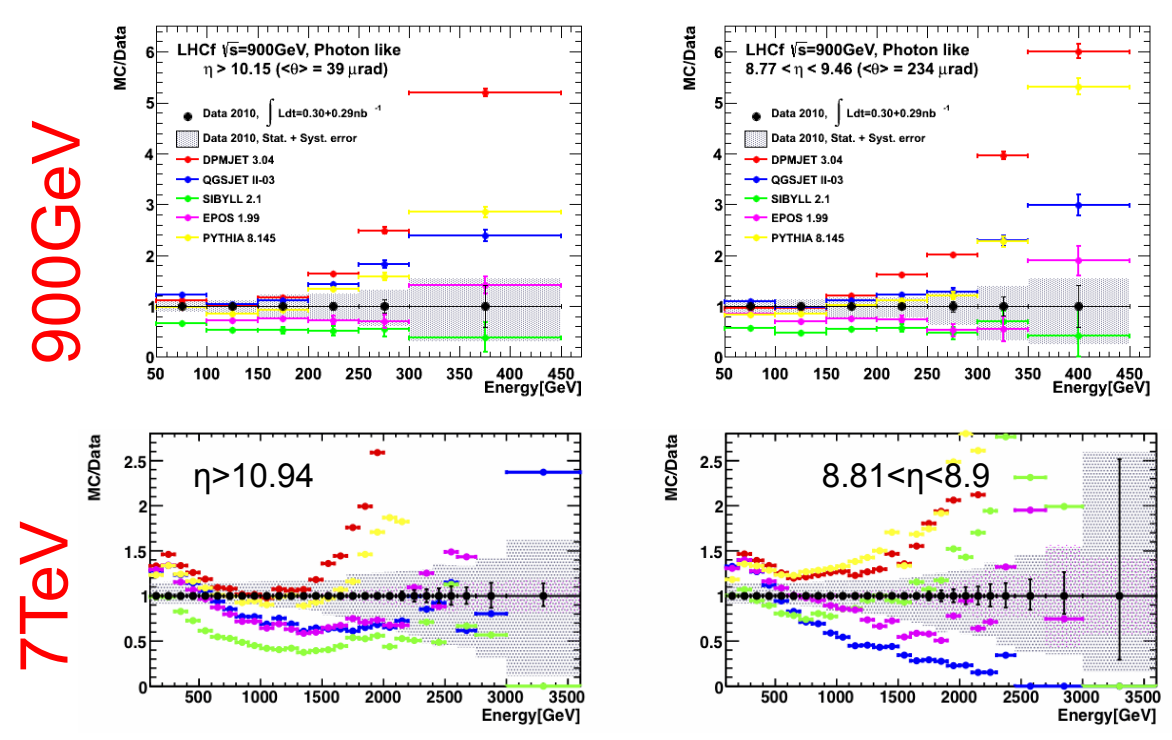

Figure 1: Single photon energy spectra measured by LHCf in $\eta>10.94$ (left) and $8.81<\eta<8.99$ (right) bin, respectively, for $7 \mathrm{TeV}$ (top panel) and $\eta>10.15$ (left) and $8.77<\eta<9.46$ (right) bin, respectively, for $900 \mathrm{GeV}$ (bottom panel) p-p collisions. The ratio of MC results predictions for DPMJET III 3.04 (red), QGSJET II-03 (blue), SIBYLL 2.1 (green), EPOS 1.99 (magenta) and PYTHIA 8.145 (yellow) to experimental data are shown in the plots. Error bars and gray shaded areas in each plot indicate the statistical and the systematic errors, respectively. Figures from Ref. [7, 8].

\subsection{Measurement of the neutral pion transverse momentum spectra}

The measurement of the single photon energy spectra reported in the previous section are very important to constrain the interaction models used in HECR but are not the only important measurement. A deeper insight can be achieved by measuring the transverse momentum spectra of photons and $\pi^{0}$. For this reason, in addition to the measurement of the single photon spectra, the LHCf experiment has recently finalised the measurement of the transverse momentum spectra for different rapidity bins for $\pi^{0}$ produced in $7 \mathrm{TeV}$ p-p collisions at LHC. The integrated luminosities corresponding to the data used in this analysis are $2.53 \mathrm{nb}^{-1}$ (Arm1) and $1.90 \mathrm{nb}^{-1}$ (Arm2) after the data taking live times were taken into account. The $\pi^{0}$ are reconstructed in LHCf through the identification of their decays in two photons. Events are selected requiring that the two photons enter different calorimeter towers; due to the geometrical acceptance of the detector only photons from $\pi^{0}$ decays with an opening angle of $\theta<0.4 \mathrm{mrad}$ can be detected. Energy, $\mathrm{p}_{\mathrm{T}}$ and rapidity of the $\pi^{0}$ are reconstructed through the measurement of the photon energy and incident position in each calorimeter. In order to ensure good event reconstruction efficiency and geometrical acceptance, the range of the $\pi^{0}$ rapidity and transverse momentum are limited to $8.9<y<11.0$ and $p_{\mathrm{T}}<0.6 \mathrm{GeV} / \mathrm{c}$, respectively. Figure 2 shows the ratios of $\mathrm{p}_{\mathrm{T}}$ spectra predicted by DPMJET 3.04 (solid, red), QGSJET II-03 (dashed, blue), SIBYLL 2.1 (dotted, green), EPOS 1.99 (dashed dotted, 
magenta), and PYTHIA 8.145 (default parameter set, dashed double-dotted, brown) to the combined ARM1 and ARM2 $p_{\mathrm{T}}$ spectra (black dots). Error bars have been taken from the statistical and systematic uncertainties. Among hadronic interaction models tested in this analysis, EPOS 1.99 shows the best overall agreement with the LHCf data, although it behaves softer than the data in the low $\mathrm{p}_{\mathrm{T}}$ region, $\mathrm{p}_{\mathrm{T}} \lesssim 0.4 \mathrm{GeV} / \mathrm{c}$ in $9.0<y<9.4$ and $\mathrm{p}_{\mathrm{T}} \lesssim 0.3 \mathrm{GeV} / \mathrm{c}$ in $9.4<y<9.6$, and behaves harder in the large $\mathrm{p}_{\mathrm{T}}$ region. DPMJET 3.04 and PYTHIA 8.145 show overall agreement with the LHCf data for $9.2<y<9.6$ and $\mathrm{p}_{\mathrm{T}}<0.25 \mathrm{GeV} / \mathrm{c}$, while the expected $\pi^{0}$ productions rates by both models exceed the LHCf data for larger $\mathrm{p}_{\mathrm{T}}$. Also SIBYLL 2.1 predicts harder pion spectra than the LHCf data, although the expected $\pi^{0}$ yield is generally small. Finally, QGSJET II-03 predicts $\pi^{0}$ spectra that are softer than the LHCf data and the other models.
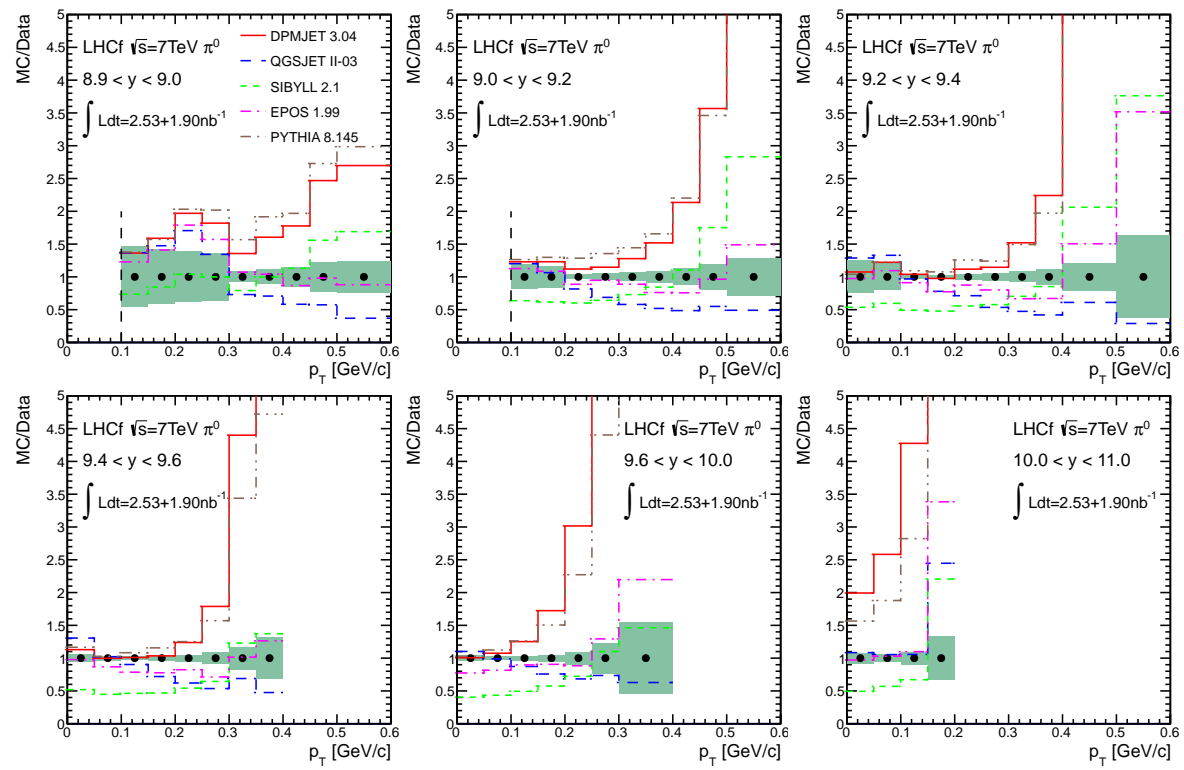

Figure 2: Ratio of the combined ARM1 and ARM2 $\pi^{0} \mathrm{p}_{\mathrm{T}}$ spectra to the $\mathrm{p}_{\mathrm{T}}$ spectra predicted by various hadronic interaction models. Shaded areas indicate the range of total uncertainties of the combined spectra. Figure from Ref. [9].

By fitting the $\mathrm{p}_{\mathrm{T}}$ spectra in each rapidity bin it is possible to extract the average transverse momentum, $\left\langle\mathrm{p}_{\mathrm{T}}>\right.$, which results to be consistent with typical values for soft QCD processes. Comparison between the LHCf and UA7 [5] results indicate an $\left\langle\mathrm{p}_{\mathrm{T}}>\right.$ versus rapidity that is independent of the center of mass energy, in agreement with the expectation of EPOS 1.99, while SYBILL 2.1 tipically gives harder $\pi^{0}$ spectra, namely larger $<\mathrm{p}_{\mathrm{T}}>$, and QGSJET II-03 gives softer $\pi^{0}$ spectra, namely smaller $<\mathrm{p}_{\mathrm{T}}>$ than the experimental data.

\subsection{Neutron spectra}

The analysis workflow for the neutron energy spectra measurement is similar to the workflow used for the $\gamma$ analysis, described in [7]. The luminosity has been measured with the help of the LHCf front counter rates, properly normalized with the Van der Meer LHC scan. Particle identification has been carried out by looking at the longitudinal shower development, using two dimensional cuts in the L20\% and L90\% plane, where L20\% and L90\% are the longitudinal depths 
containing $20 \%$ and $90 \%$ of the total deposited energy, respectively. Hit position has been evaluated by using the transverse shower distribution, measured with the position sensitive layers, to optimally correct the energy measured for the leakage effects.

Figure 3 shows the preliminary energy spectra of forward neutrons measured by the Arm 1 detector compared with the MC predictions; left and right panels correspond to the spectra measured on the small and large tower, respectively. The effect of the limited energy resolution for hadrons
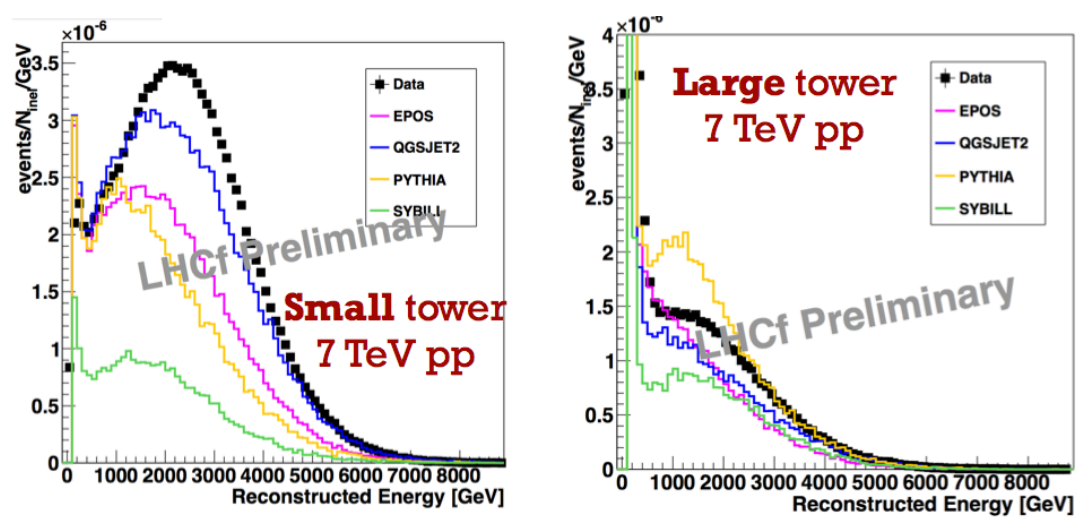

Figure 3: Preliminary neutron spectra measured by LHCf in the $\sqrt{s}=7 \mathrm{TeV}$-p collisions in the small and large tower.

$(\simeq 30 \%)$ is evident from these plots. A detailed unfolding procedure, currently underway, is hence necessary to disentangle the detector effects from the real differences in the spectra expected from the various models. Despite the limited energy resolution achievable, the neutron spectra are of fundamental importance to better understand the muon excess problem reported by both Auger [15] and TA [16]. This excess could be indeed interpreted in terms of an underestimation of the baryon production in the hadronic interaction models used to derive the CR spectra [17].

\section{Preliminary results in $\mathrm{p}-\mathrm{Pb}$ collisions}

At the beginning of 2013, LHCf took data in the p-Pb collisions at a center-of-mass energy of $\sqrt{s_{N N}}=5 \mathrm{TeV}$. Due to the higher multiplicity expected in ion collisions, only the ARM2 detector, which has a higher granularity thanks to the silicon microstrip layers, was used during the $\mathrm{p}-\mathrm{Pb}$ run. In most of the operation time, the detector was located on the p-remnant side. The physics aim of the $\mathrm{p}-\mathrm{Pb}$ measurement is the investigation of the nuclear effect on the production of forward energetic particles, that is one of the important effects for modelling the interactions between cosmic-rays and atmosphere. The run was very successful, and the quality of data excellent, as demonstrated by the very clean $\pi^{0}$ invariant mass peak shown in Figure 4.

The analysis of $\mathrm{p}-\mathrm{Pb}$ data to obtain $\gamma, \pi^{0}$ and neutron transverse momentum spectra in different rapidity bins and their impact point distributions is underway.

\section{Future activities and summary}

In addition to the ongoing analysis activities, the LHCf Collaboration is working on the up- 


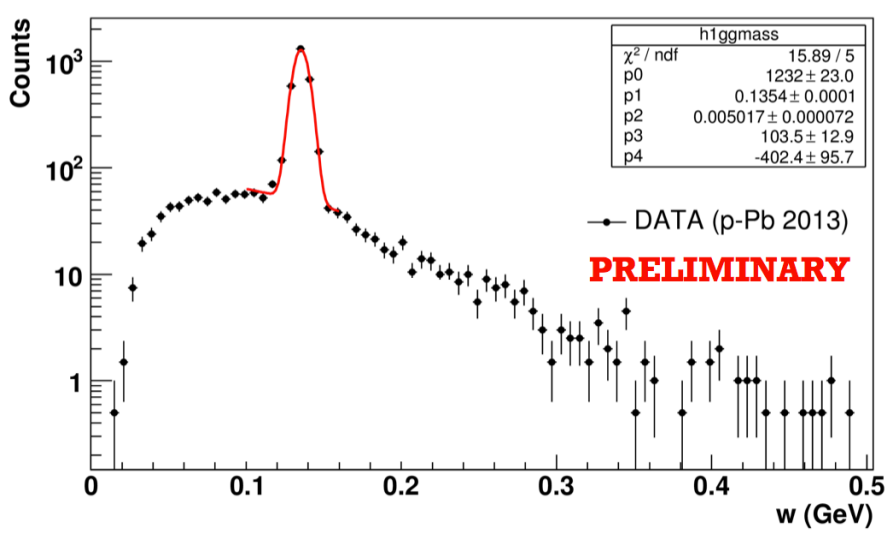

Figure 4: Invariant mass spectra reconstructed in the $\mathrm{p}-\mathrm{Pb}$ collisions in the proton remnant side.

grade of the detector to improve the radiation resistance in view of the $14 \mathrm{TeV}$ p-p run, currently foreseen in 2015. The re-installation of the detector is currently scheduled at the end of 2014. The upgraded LHCf calorimeters will foresee GSO slabs instead of scintillators for both ARM1 and ARM2 and also additional improvements in the front-end electronics of the silicon position sensitive layers of ARM2 detectors as well as an optimization of the layout to improve the stand-alone silicon energy resolution.

\section{References}

[1] M. Takeda et al., Phys. Rev. Lett. 81 (1998) 1163.

[2] R. U. Abbasi et al., Phys. Rev. Lett. 92 (2004) 1511.

[3] J. R. T. de Mello Neto [Pierre Auger Collaboration], Adv. Space Res. (2013)

[4] P. Sokolsky [TA and HiRes Collaboration], EPJ Web Conf. 52 (2013) 06002.

[5] E. Paré et al., Phys. Lett. B 242 (1990) 531.

[6] O. Adriani et al., JINST 3 (2008) S08006.

[7] O. Adriani et al., Phys. Lett. B703 (2011) 128.

[8] O. Adriani et al., Phys. Lett. B715 (2012) 298.

[9] O. Adriani et al., Phys. Rev. D 86 (2011) 092001.

[10] F.W. Bopp et al., Phys. Rev. C77 (2008) 014904.

[11] S. Ostapchenko, Phys. Rev. D83 (2011) 014108.

[12] E.-J. Ahn et al., Phys. Rev. D80 (2009) 094003.

[13] K. Werner et al., Nucl.Phys.Proc.Suppl. 175-176 (2008) 81.

[14] T. Sjöstand, et al., Comput. Phys. Comm. 178 (2008) 852.

[15] P. Abreu et al. [The Pierre Auger Collaboration], Proc. of 32th Int. Cosmic Ray Conf., Beijing (2011), [arXiv:1107.4804 [astro-ph.HE]].

[16] T. Abu-Zayyad, et al. [TA Collaboration], Astrophys. J. 768 (2013) L1

[17] T. Pierog and K. Warner, Phys. Rev. Lett. 101 (2008) 171101. 